Agron. Mesoam. 29(2):315-323. Mayo-agosto, 2018

ISSN 2215-3608, doi:10.15517/ma.v29i2.27126

http://www.revistas.ucr.ac.cr/index.php/agromeso

\title{
Curva de crecimiento poblacional de Spodoptera frugiperda en maíz en Irapuato, Guanajuato, México ${ }^{1}$
}

\section{Population growth curve of Spodoptera frugiperda in maize in Irapuato, Guanajuato, Mexico}

\author{
Oscar Alejandro Martínez-Jaime ${ }^{2}$, Manuel Darío Salas-Araiza ${ }^{2,3}$, José Antonio Díaz-García ${ }^{4}$
}

\begin{abstract}
Resumen
El gusano cogollero Spodoptera frugiperda Smith (Lepidoptera: Noctuidae) es una plaga importante del maíz (Zea mays L.) en Irapuato, Guanajuato, México. El objetivo del estudio fue obtener la curva de crecimiento poblacional (y su estimación) de los adultos de S. frugiperda en el cultivo del maíz en Irapuato, Guanajuato, México. Mediante la técnica de regresión múltiple, se obtuvo la forma funcional de un polinomio de tercer grado en términos de la temperatura y precipitación, con promedios mensuales disponibles de 1993 a 2002. La ecuación estimada fue: $\mathrm{Y}=821,347-$ $104,728 \mathrm{~T}+2,737 \mathrm{P}+3,448 \mathrm{~T}^{2}-0,019 \mathrm{P}^{2}-0,180 \mathrm{TP}+0,001 \mathrm{TP}^{2}(1)$, donde $\mathrm{T}$ fue el promedio de la temperatura media mensual, $\mathrm{P}$ fue el promedio de la precipitación mensual acumulada y $\mathrm{Y}$ fue el número promedio por mes de adultos de $S$. frugiperda. Además, utilizando técnicas estándar de programación matemática, se calculó el punto crítico de temperatura y precipitación $\left(\mathrm{T}=13,70^{\circ} \mathrm{C}, \mathrm{P}=38,70 \mathrm{~mm}\right)$, para el cual se obtuvo el número promedio mensual máximo de individuos de esta especie, que fue de 39 adultos. El modelo de predicción (1) aportó información para tener mayor conocimiento sobre la fluctuación poblacional de esta especie, contribuyendo por lo tanto a hacer más eficiente el manejo integrado de esta plaga en el cultivo de maíz en la región de "El Bajío" de Guanajuato, México.
\end{abstract}

Palabras clave: modelos de simulación, fenología, clima, dinámica de poblaciones, fitófagos.

\begin{abstract}
The fall armyworm Spodoptera frugiperda Smith (Lepidoptera: Noctuidae) is an important pest of maize (Zea mays L.) in Irapuato, Guanajuato, Mexico. The objective of the study was to obtain the population growth curve (and its estimation) of the adults of S. frugiperda in the maize crop in Irapuato, Guanajuato, Mexico. Applying the multiple regression technique, the functional form of a third-degree polynomial was obtained, in terms of temperature and precipitation, using the monthly averages available from 1993 to 2002. The estimated equation was: $\mathrm{Y}=$ $821.347-104.728 \mathrm{~T}+2.737 \mathrm{P}+3.448 \mathrm{~T}^{2}-0.019 \mathrm{P}^{2}-0.180 \mathrm{TP}+0.001 \mathrm{TP}^{2}(1)$, where $\mathrm{T}$ was the average monthly mean temperature, $\mathrm{P}$ was the average monthly accumulated precipitation, and $\mathrm{Y}$ was the average of adults of $S$. frugiperda per month. In addition, using standard mathematical programming techniques, the critical temperature and
\end{abstract}

1 Recibido: 28 de marzo, 2017. Aceptado: 3 de julio, 2017. Este trabajo formó parte del proyecto "Enemigos naturales de Spodoptera frugiperda y Helicoverpa zea como una alternativa al uso de insecticidas" financiado por la Universidad De La Salle Bajío, y se llevó a cabo en la Universidad de Guanajuato, en Irapuato Guanajuato, México.

2 Universidad de Guanajuato, Km. 9 carretera Irapuato-Silao, C.P. 36821, Irapuato, Guanajuato, México. oscarja@ugto.mx

3 Universidad de La Salle, Bajío. Av. Universidad 602, Lomas del Campestre, C.P. 37150, León, Guanajuato, México. salasm@ugto.mx

4 Universidad Autónoma de Chihuahua, Facultad de Zootecnia y Ecología, Periférico Francisco R. Almada Km. 1, Col. Zootecnia, C.P. 33820, Chihuahua, Chihuahua, México.jadiaz@uach.mx 
the critical precipitation were calculated $\left(\mathrm{T}=13.70{ }^{\circ} \mathrm{C}, \mathrm{P}=38.70 \mathrm{~mm}\right)$ obtaining the average of maximum number of individuals of this specie per month, which was 39 adults. The prediction model (1) provided information to reach deep understanding of the fluctuation of this species population, and contributed, therefore, to make the integrated pest management in maize crop more efficient, in the region of "El Bajío" of Guanajuato.

Keyword: simulation models, phenology, weather, population dynamics, phytophagous.

\section{Introducción}

El gusano cogollero $S$. frugiperda Smith (Lepidoptera: Noctuidae) reduce el rendimiento del maíz en 30\% en México, pero en ocasiones causa pérdida total (Aguirre et al., 2016). Es una especie polífaga que se alimenta de varias familias vegetales (Cruz et al., 2012), es de origen tropical y su distribución ocurre a lo largo del continente americano (Meagher y Nagoshi, 2004). S. frugiperda tiene hábitos migratorios (Nagoshi y Meagher, 2008), pero también puede estar presente durante todo el año en la misma localidad, desarrollándose en cultivos alternos como alfalfa, arroz, algodón, etc. (Casmuz et al., 2010). En el estado de Guanajuato ocasiona severos daños al maíz y sorgo, ya que se alimentan del verticilo (Salas et al., 2002). Durante el día, los adultos permanecen entre hojarasca, maleza o en sitios sombreados, y durante la noche se vuelven activos. Son atraídos por la luz y se pueden desplazar a varios kilómetros de distancia (Negrete y Morales, 2003).

Entender la relación entre los diferentes regímenes de temperatura con los parámetros del ciclo de vida, como son los días de desarrollo larvario y la época de vuelo de los adultos, son esenciales para formular modelos de dinámica poblacional (Auad et al., 2015). El periodo de vuelo estacional de un insecto es una herramienta que ayuda a planear las estrategias de control necesarias para evitar pérdidas económicas en los cultivos (Klingeman et al., 2015).

Los modelos matemáticos que aproximan adecuadamente las poblaciones de insectos, con frecuencia incluyen factores externos e internos que inciden en la fluctuación de poblaciones a través del tiempo (Venette et al., 2010). Se ha probado que las variables climáticas influyen en la supervivencia y duración de los ciclos de vida de los insectos, causando variaciones en el número de individuos (Hodgson et al., 2011). La temperatura es el factor abiótico que más afecta el desarrollo de los insectos y su dinámica poblacional, por lo que, la selección adecuada del modelo matemático que describa esta relación, es fundamental en el manejo de plagas.

Se han obtenido modelos para estimar el número de insectos a través de la técnica de regresión múltiple, donde las variables independientes han sido algunas características del clima; por ejemplo, la regresión lineal constituye una herramienta para modelar el desarrollo de Plutella xylostella (Lepidoptera: Yponomeutidae), en función de la temperatura (Marchioro y Foerster, 2011). El modelo ajustado con información de tres años, determinó que la distribución espacial de Circulifer tenellus (Hemiptera: Cicadellidae) depende de la temperatura media, la precipitación y la velocidad del viento (Murphy et al., 2012). En otro modelo obtenido con la misma técnica y con datos de un año, se concluyó que la temperatura y la precipitación explican el 89\% de la variación en el número de individuos de Dendroctonus mexicanus (Coleoptera: Scolytidae) (Villa y Villa, 1996). En este mismo coleóptero, se propuso una ecuación con buen ajuste, para explicar la relación entre la abundancia de individuos con la temperatura y la precipitación pluvial (Cuéllar-Rodríguez et al., 2012).

Con el empleo de la regresión múltiple se estimó la densidad poblacional de curculiónidos en alfalfa, en términos de la temperatura, la precipitación y el fotoperiodo; se obtuvieron coeficientes de determinación aceptables (Gopar y Ves-Losada, 2004). Otro modelo de regresión múltiple de buen ajuste, fue desarrollado para estimar la población de adultos de Diabrotica balteata (Coleoptera: Chrysomelidae) a partir de variables climáticas, 
la función obtenida se utilizó como una herramienta de pronóstico que permitió mejorar el manejo de esta plaga (Rodríguez-Del-Bosque y Magallanes-Estala, 1994).

Dada la importancia de S. frugiperda en el cultivo del maíz en la región de "El Bajío", es necesario obtener información sobre su fluctuación poblacional, la cual se afecta principalmente por la temperatura y la precipitación (Murúa et al., 2006). Con el fin de buscar estrategias para contribuir al manejo integrado de este nóctuido en maíz, se planteó este trabajo con el objetivo de obtener la forma funcional de la curva de crecimiento poblacional (y su estimación) de los adultos de S. frugiperda en el cultivo del maíz en Irapuato, Guanajuato, México.

\section{Materiales y métodos}

El presente estudio se realizó del 1 de enero de 1993 al 31 diciembre de 2002, en el campo experimental de la División de Ciencias de la Vida (DICIVA) de la Universidad de Guanajuato (UG), ubicado en la Ex-Hacienda "El Copal", en Irapuato, Guanajuato, México. Este lugar se encuentra a $1757 \mathrm{msnm}$, y se localiza a 2044' 39" N y 101 19' 39" O; con clasificación de clima BS(hw)(h)(e) semicálido subhúmedo con lluvias en verano (García, 2004), una temperatura media anual de $19,40{ }^{\circ} \mathrm{C}$ y una precipitación pluvial acumulada anual de $530,90 \mathrm{~mm}$ (CONAGUA, 2015).

Las palomillas de $S$. frugiperda se recolectaron mediante trampa de luz negra de 15 watts (Cantelo, 1990); se instaló una trampa al inicio de cada mes en el borde de una parcela de alrededor de cuatro hectáreas, en la cual había maíz y otros cultivos como trigo, cuando el maíz era cosechado, al inicio de cada mes, se cambiaba la trampa, sumando doce trampas por año. Los insectos capturados en el recipiente de la trampa, se contaron semanalmente en el Laboratorio de Entomología de la DICIVA-UG, y se registró el total mensual (suma de cuatro semanas) de ejemplares adultos, durante los diez años que abarcó este trabajo. La información climática (temperatura media y precipitación pluvial acumulada), se obtuvo de la estación meteorológica "El Copal", contigua al sitio de muestreo.

Se consideró como variable dependiente el promedio mensual de individuos de $S$. frugiperda, el cual se calculó con las observaciones totales mensuales efectuadas durante diez años, y como variables independientes los promedios mensuales de temperatura media (T) y de precipitación pluvial acumulada (P) de 1993 a 2002. Se procedió a estimar la curva de crecimiento poblacional, a través de la técnica de regresión múltiple, para aproximar mediante una forma funcional el modelo de mejor ajuste, por último, se determinó el punto crítico de temperatura y precipitación ( $\mathrm{T}, \mathrm{P})$, que produce el número máximo (promedio mensual) de individuos de esta especie, este valor extremo para la función de dos variables, se calculó aplicando un procedimiento de optimización perteneciente a la familia de los métodos quasi-Newton que aproxima el algoritmo de Broyden-Fletcher-Goldfarb-Shanno (BFGS), el cual se encuentra implementado en el paquete de programación R (R Development Core Team, 2008).

\section{Resultados}

La fluctuación poblacional del número total mensual de adultos de $S$. frugiperda durante los 120 meses de evaluación, mostró irregularidad de un ciclo anual a otro (Figuras 1 y 2), excepto los últimos tres años en que los ciclos mostraron un patrón que se volvió uniforme; a mediados de 1994 y de 1995 se observó un bajo número de individuos, con un repunte mayor en 1996, año en que se registró el mayor número de palomillas. De manera sistemática, el número de adultos capturados aumentó en los meses de mayo y junio de todos los años (Figuras 1 y 2), mientras que, en términos generales, en septiembre la captura disminuyó, lo cual coincidió con una reducción en la temperatura y con la etapa en que el maíz se encuentra en la fase de maduración del grano.

La curva de crecimiento poblacional de S. frugiperda (1) y su gráfico (Figura 3), se obtuvieron con base en el paquete de programación R (R Development Core Team, 2008). Para tal efecto, se propusieron varias formas 


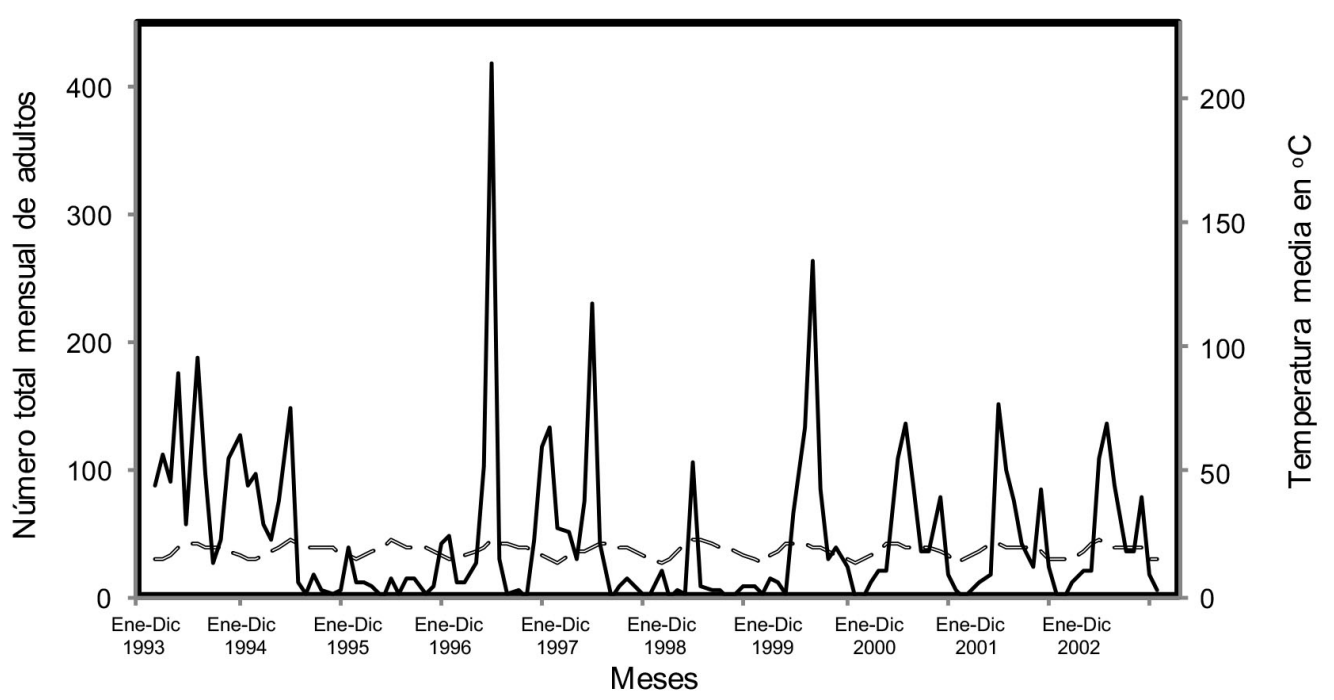

Figura 1. Fluctuación poblacional mensual de adultos de Spodoptera frugiperda, y variación de la temperatura media mensual de 1993 a 2002, en el cultivo del maíz en el campo experimental de la División de Ciencias de la Vida (DICIVA) de la Universidad de Guanajuato (UG), Irapuato, Guanajuato, México.

Figure 1. Monthly population fluctuation of adults of Spodoptera frugiperda, and variation of average monthly mean temperature from 1993 to 2002, in the maize crop at the Experimental Agricultural Field of the Life Sciences Division (DICIVA) of the University of Guanajuato (UG), Irapuato, Guanajuato, Mexico.

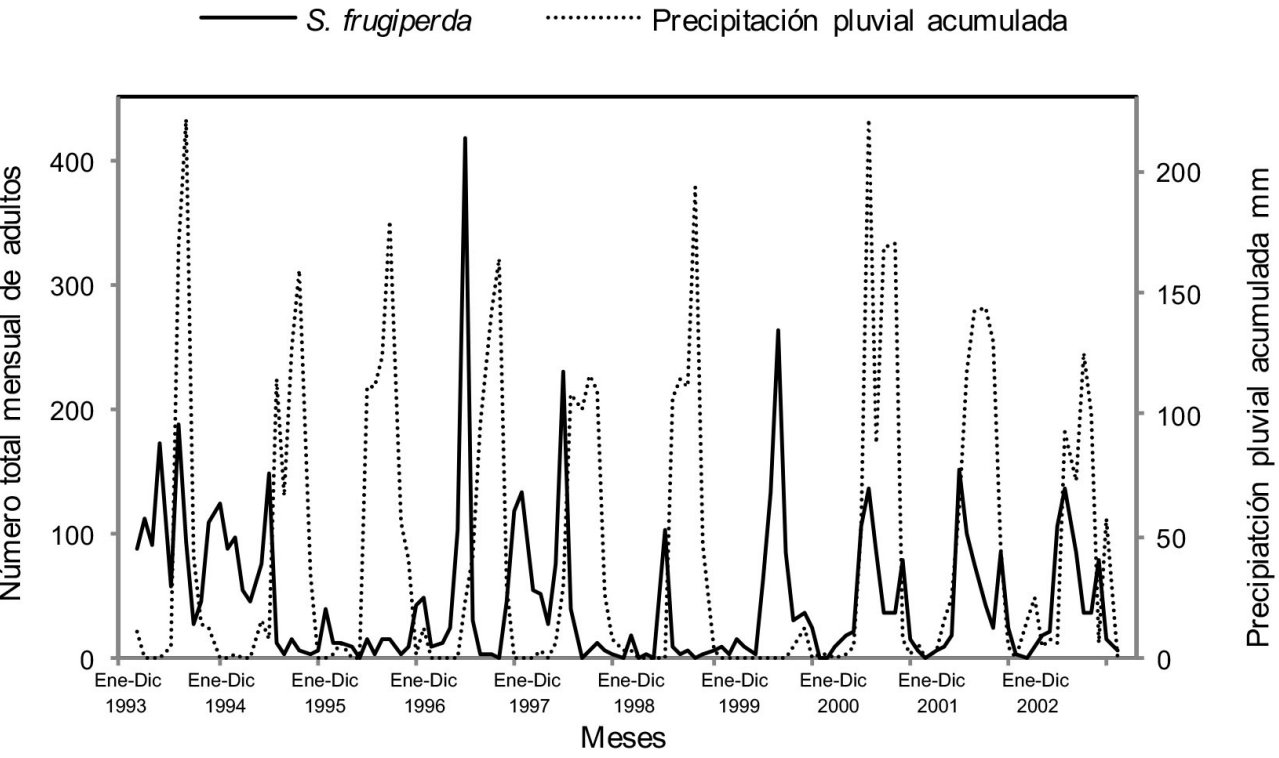

Figura 2. Fluctuación poblacional mensual de adultos de Spodoptera frugiperda, y variación de la precipitación pluvial acumulada mensual de 1993 a 2002, en el cultivo del maíz en el campo experimental de la División de Ciencias de la Vida (DICIVA) de la Universidad de Guanajuato (UG), Irapuato, Guanajuato, México.

Figure 2. Monthly population fluctuation of adults of Spodoptera frugiperda, and variation of monthly accumulated precipitation from 1993 to 2002, in the maize crop at the Experimental Agricultural Field of the Life Sciences Division (DICIVA) of the University of Guanajuato (UG), Irapuato, Guanajuato, Mexico. 
funcionales para el modelo de crecimiento, tales como el modelo lineal y el polinomial de varios grados. Luego, se empleó como medida de bondad de ajuste, el valor del coeficiente de determinación $\left(\mathrm{R}^{2}=0,87\right)$, se logró seleccionar el modelo de mejor ajuste, con una función polinómica de tercer grado definida por:

$$
\mathrm{Y}=821,347-104,728 \mathrm{~T}+2,737 \mathrm{P}+3,448 \mathrm{~T}^{2}-0,019 \mathrm{P}^{2}-0,180 \mathrm{TP}+0,001 \mathrm{TP}^{2},
$$

donde $\mathrm{T}$ fue el promedio de la temperatura media mensual, $\mathrm{P}$ el promedio de la precipitación mensual acumulada y Y el número promedio mensual de palomillas durante 1993 a 2002. Los criterios de selección de este modelo fueron los valores del estadístico $\mathrm{F}=5,77$ y su probabilidad $0,0369 *$ del análisis de varianza de la regresión, así como el valor del coeficiente de determinación $\left(\mathrm{R}^{2}=0,87\right)$ como medida de bondad de ajuste; con base en ello, se consideró esta curva de crecimiento como un modelo adecuado de estimación. El valor extremo para la función de dos variables obtenida, se calculó aplicando un procedimiento de optimización perteneciente a la familia de los métodos quasi-Newton que aproxima el algoritmo de Broyden-Fletcher-Goldfarb-Shanno (BFGS), el cual se encuentra implementado en el paquete de programación R (R Development Core Team, 2008); resultando el punto crítico $\left(\mathrm{T}=13,7^{\circ} \mathrm{C}, \mathrm{P}=38,7 \mathrm{~mm}\right.$ ) para el cual se obtuvo el número máximo de individuos adultos de esta especie, cuyo promedio mensual fue de 39 palomillas.

\section{Discusión}

La presente investigación mostró cómo la fluctuación poblacional de $S$. frugiperda varió a lo largo de cada año, y esta a su vez, fue diferente entre los años de muestreo (Figuras 1 y 2), coincidiendo con un estudio similar realizado en Yucatán, México, donde no hubo un esquema estacional reproducible anualmente, cuando se capturaron palomillas de S. frugiperda en trampas con feromona sexual en el periodo de 2001 a 2003 (NexticapanGarcéz et al., 2009). Esta especie es de hábitos migratorios y se caracteriza por presentar una amplia variación anual cuando se muestrea con trampa de luz, y por tener incrementos notables en sus poblaciones luego de varios años (Chapman y Lienk, 1981), tal como se pudo observar en los resultados de este trabajo, al presentar poblaciones mayores durante 1996, 1997 y 1999.

La aparición de poblaciones de insectos puede ocurrir de manera extensa y persistir por periodos de tiempo cortos, pudiendo ser cíclicos o irregulares, y su fluctuación depende principalmente de las condiciones ambientales; por ejemplo, las poblaciones pueden aumentar después de un periodo de sequía, ya que, al incrementarse la concentración de los contenidos nutricionales en las plantas, se satisfacen más fácilmente los requerimientos alimenticios del insecto (Coyle et al., 2013). En condiciones naturales los insectos se encuentran expuestos a ciclos variables de temperatura, pero cuando las condiciones térmicas son constantes, el desarrollo de los insectos es diferente, ya que algunos muestran un ciclo de crecimiento más corto cuando la temperatura fluctúa, que cuando permanece uniforme (Auad et al., 2015). En un estudio relacionado con Helicoverpa armigera (Lepidoptera: Noctuidae), se observó que su desarrollo fue más rápido cuando la temperatura varió alrededor de $25{ }^{\circ} \mathrm{C}$, que cuando la temperatura permaneció en $25^{\circ} \mathrm{C}$ (Mironidis y Savopoulou-Soultani, 2008), lo que posiblemente ayude a explicar la variación poblacional observada a lo largo de esta investigación, provocada, tal vez, por los cambios de temperatura.

En la presente investigación, el máximo número de palomillas capturadas se relacionó con una precipitación mensual acumulada de $38,70 \mathrm{~mm}$; esto es importante en insectos que pupan en el suelo, ya que puede usarse en el manejo integrado, para pronosticar cuando se tendrá la máxima emergencia de adultos. Sin embargo, como se puede ver con los resultados obtenidos, no hubo un claro comportamiento migratorio, ya que a lo largo del año hubo capturas de adultos, posiblemente por las condiciones favorables de clima presentes en la región y la disponibilidad de hospederos alternos. 
El coeficiente de determinación del modelo estimado en este estudio $\left(R^{2}=0,87\right)$ fue mayor que los obtenidos en los modelos propuestos para D. mexicanus $\left(\mathrm{R}^{2}=0,64\right)$ (Cuéllar-Rodríguez et al., 2012), A. tessellatus $\left(\mathrm{R}^{2}=0,67\right)$ (Gopar y Ves-Losada, 2004) y D. balteata $\left(\mathrm{R}^{2}=0,86\right)$ (Rodríguez-Del-Bosque y Magallanes-Estala, 1994), lo que demostró un mejor ajuste en el modelo obtenido.

El promedio mensual de la temperatura media obtenida en este trabajo fue de $13,70{ }^{\circ} \mathrm{C}$, el cual proporcionó la cantidad más alta de adultos de gusano cogollero capturados, y fue semejante a la temperatura obtenida en una investigación realizada en Perú para conocer la fluctuación poblacional de Spodoptera spp., usando trampas de saco negro, donde los adultos proliferaron cuando el promedio entre las temperaturas mínima y máxima estuvo por encima de $\operatorname{los} 10^{\circ} \mathrm{C}$ (Neyra y Chanducas, 2009). La fluctuación de la temperatura influye en el desarrollo de los insectos, sobre todo en ciertos procesos fisiológicos en los que intervienen enzimas, los cuales son favorecidos por las variaciones de temperatura (Rock, 1985). Asimismo, se ha observado que el ciclo de desarrollo de P. xylostella se acorta cuando la temperatura es variable, lo cual fue asociado con el incremento de la regulación de diversas proteínas que juegan un papel esencial en el metabolismo energético y la degradación de estas (Bahar et al., 2012).

El trabajo no pretende predecir la incidencia de la plaga a través del tiempo, los resultados obtenidos solo explican el comportamiento de las poblaciones de $S$. frugiperda en términos de las condiciones climáticas, mediante la estimación de la curva de crecimiento poblacional. Los valores de temperatura y precipitación para los cuales se presentó el mayor número de palomillas capturadas, hace suponer que su actividad (periodo de vuelo) es mayor en estas condiciones, factor importante al momento de orientar estrategias en el manejo integrado de plagas (Klingeman et al., 2015), ya que se puede realizar la liberación de organismos benéficos, o hacer aplicaciones oportunas de insecticidas, para evitar pérdidas de rendimiento en el cultivo de maíz en la región de "El Bajío" guanajuatense, sobre todo en agricultura de temporal.

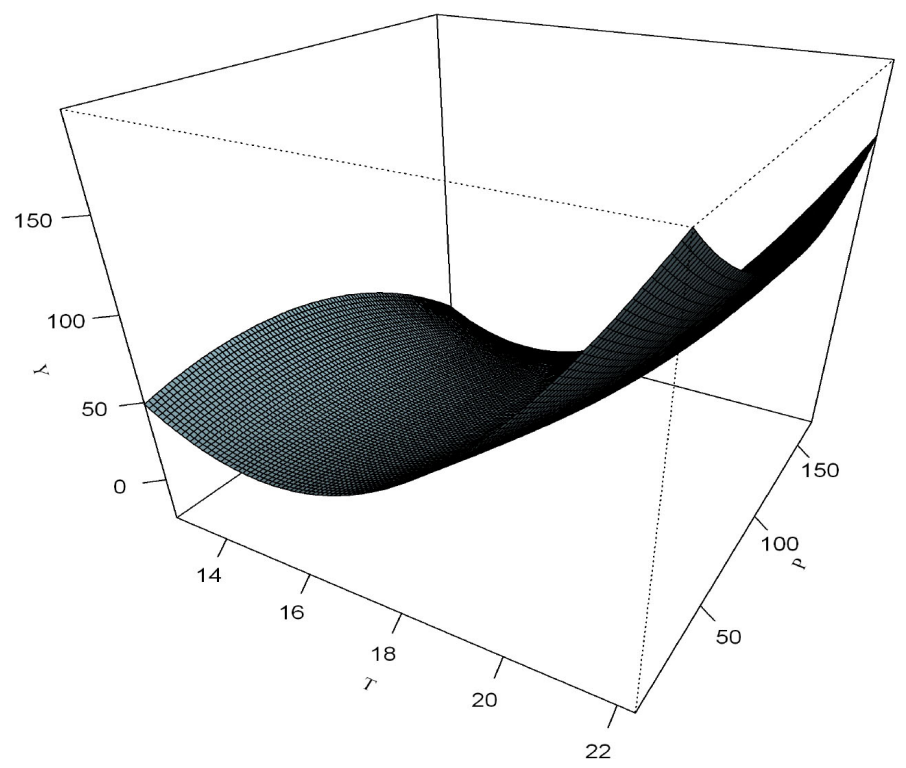

Figura 3. Curva de crecimiento estimada para el número de adultos de Spodoptera frugiperda (Y) en términos de la temperatura (T) y la precipitación (P) en el cultivo de maíz, para el periodo 1993 a 2002, en el campo experimental de la División de Ciencias de la Vida (DICIVA) de la Universidad de Guanajuato (UG), Irapuato, Guanajuato, Mexico.

Figure 3. Estimated growth curve for adults of Spodoptera frugiperda $(\mathrm{Y})$ in terms of temperature $(\mathrm{T})$ and precipitation $(\mathrm{P})$ in maize crop, from 1993 to 2002, at the Experimental Agricultural Field of the Life Sciences Division (DICIVA) of the University of Guanajuato (UG), Irapuato, Guanajuato, Mexico. 


\section{Conclusiones}

Después de probar varios modelos lineales y no lineales, se empleó el criterio del mayor $\mathrm{R}^{2}$ para su selección, los resultados de este trabajo indicaron que la relación funcional del número de palomillas en términos de la temperatura y precipitación, fue un modelo polinomial de tercer grado (ecuación 1) (Figura 3). La curva de crecimiento poblacional obtenida permitió estimar la población de adultos de $S$. frugiperda en términos de la temperatura y precipitación, así como el punto crítico $\left(\mathrm{T}=13,70^{\circ} \mathrm{C}\right.$ y $\left.\mathrm{P}=38,70 \mathrm{~mm}\right)$ para el cual se presentó el máximo número de palomillas, que fue un promedio mensual de 39 adultos.

\section{Literatura citada}

Aguirre, L.A., A. Hernández-Juárez, M. Flores, E. Cerna, J. Landeros, G.A. Frías, and M.K. Harris. 2016. Evaluation of foliar damage by Spodoptera frugiperda (Lepidoptera: Noctuidae) to genetically modified corn (Poales: Poaceae) in Mexico. FL Entomol. 99:276-280. doi:10.1653/024.099.0218

Auad, A.M., S.E.B. Silva, J.C. Santos, and T.M. Vieira. 2015. Impact of fluctuating and constant temperatures on key life history parameters of Sipha flava (Hemiptera: Aphididae). FL Entomol. 98:424-429. doi:10.1653/024.098.0205

Bahar, M.H., J.J. Soroka, and L.M. Dosdall. 2012. Constant versus fluctuating temperatures in the interactions between Plutella xylostella (Lepidoptera: Plutellidae) and its larval parasitoid Diadegma insulare (Hymenoptera: Ichneumonidae). Environ. Entomol. 41:1653-1661. doi:10.1603/EN12156

Cantelo, W.W. 1990. Comparative efficacy of a blacklight trap and a Robinson trap in trapping moths. Southwest. Entomol. 15:159-162.

Casmuz, A., M.L. Juárez, M.G. Socías, M.G. Murúa, S. Prieto, S. Medina, E. Willink, y G. Gastaminza. 2010. Revisión de los hospederos del gusano cogollero del maíz, Spodoptera frugiperda (Lepidoptera: Noctuidae). Rev. Soc. Entomol. Argent. 69:209-231.

Chapman, P.J., and S.E. Lienk. 1981. Flight periods of adults of cutworms, armyworms loopers and others (family Noctuidae) injurious to vegetable and field crops. Agric. Bull. 14. New York State Agricultural Experiment Station, Ithaca, NY, USA.

CONAGUA (Comisión Nacional del Agua). 2015. Servicio Meteorológico Nacional: Normales climatológicas periodo 1951-2010. CONAGUA, MEX. http://smn.cna.gob.mx/tools/RESOURCES/Normales5110/NORMAL07165.TXT (consultado 30 abr. 2015).

Coyle, D.R., J. Pickering, K.A. Dyer, F.R. Lehman, J.E. Mohan, and K.J.K. Gandhi. 2013. Dynamics of an unprecedented outbreak of two native moth species, Cissusa spadix and Phoeberia atomaris (Lepidoptera: Noctuidae), on oak trees (Quercus spp.) in the Southeastern United States. Am. Entomol. 59(2):82-94.

Cruz, I., M.L. Corrêa-Figueiredo, R. Braga-da-Silva, I. Fernandes-da-Silva, C. deSouza-Paula, and J.E. Foster. 2012. Using sex pheromone traps in the decision-making process for pesticide application against fall armyworm (Spodoptera frugiperda [Smith] [Lepidoptera: Noctuidae]) larvae in maize. Int. J. Pest. Manage 58:83-90. doi:10.1080/09670874.2012.655702

Cuéllar-Rodríguez, G., A. Equihua-Martínez, E. Estrada-Venegas, T. Méndez-Montiel, J. Villa-Castillo, y J. Romero-Nápoles. 2012. Fluctuación poblacional de Dendroctonus mexicanus Hopkins (Coleoptera: Curculionidae: Scolytinae) atraídos a trampas en el noreste de México y su correlación con variables climáticas. Bol. Mus. Entomol. 13:12-19. 
García, E. 2004. Modificaciones al sistema de clasificación climática de Koppen: para adaptarlo a las condiciones de la República Mexicana. Instituto de Geografía, y UNAM, México D.F., MEX.

Gopar, A., y J.C. Ves-Losada. 2004. Estudio sobre la fluctuación poblacional de gorgojos (Coleoptera: Curculionidae) adultos que afectan a la alfalfa (Medicago sativa, L.). Publicación Técnica № 57. Instituto Nacional de Tecnología Agropecuaria, Anguil, LA Pampa, ARG.

Hodgson, J.A., C.D. Thomas, T.H. Oliver, B.J. Anderson, T.M. Brereton, and E.E. Crone. 2011. Predicting insect phenology across space and time. Glob. Change Biol. 17:1289-1300. doi: 10.1111/j.1365-2486.2010.02308.x

Klingeman, W.E., J.A. Hansen, J.P. Basham, J.B. Oliver, N.N. Youssef, W. Swink, C.A. Nalepa, D.C. Fare, and J.K. Moulton. 2015. Seasonal flight activity and distribution of metallic woodboring beetles (Coleoptera: Buprestidae) collected in North Carolina and Tennessee. FL Entomol. 98:579-587. doi: 10.1653/024.098.0230

Marchioro, C.A., and L.A. Foerster. 2011. Development and survival of the diamondback moth, Plutella xylostella (L.) (Lepidoptera: Yponomeutidae) as a function of temperature: Effect on the number of generations in tropical and subtropical regions. Neotrop. Entomol. 40:533-541. doi: 10.1590/S1519-566X2011000500003

Meagher, R.L., and R.N. Nagoshi. 2004. Population dynamics and occurrence of Spodoptera frugiperda host strains in southern Florida. Ecol. Entomol. 29:614-620. doi:10.1111/j.0307-6946.2004.00629.x

Mironidis, G.K., and M. Savopoulou-Soultani. 2008. Development, survivorship, and reproduction of Helicoverpa armigera (Lepidoptera: Noctuidae) under constant and alternating temperatures. Environ. Entomol. 31:16-28. doi:10.1603/0046225X(2008)37[16:DSAROH]2.0.CO;2

Morales, A.J. 2000. Manejo del gusano cogollero del maíz utilizando extractos de plantas. http://www.turipana.org (consultado 29 de abr. 2015).

Murphy, A.F., S.I. Rondon, and A.S. Jensen. 2012. Population dynamics of the beet leafhopper (Hemiptera: Cicadellidae) in the Columbia Basin as influenced by abiotic variables. Environ. Entomol. 41:768-775. doi: 10.1603/EN12033

Murúa, G., J. Molina-Ochoa, and C. Coviella. 2006. Population dynamics of the fall armyworm, Spodoptera frugiperda (Lepidoptera: Noctuidae) and its parasitoids in northwestern Argentina. FL Entomol. 89:175-182. doi:10.1653/00154040(2006)89[175:PDOTFA]2.0.CO;2

Nagoshi, R.N., and R.L. Meagher. 2008. Review of fall armyworm (Lepidoptera: Noctuidae) genetic complexity and migration. FL Entomol. 91:546-554. doi:10.1653/0015-4040-91.4.546

Negrete, B.F., y J.G. Morales. 2003. El gusano cogollero del maíz (Spodoptera frugiperda Smith). Corpoica. Pronatta. 26 p. Montería, Colombia. http://bibliotecadigital.agronet.gov.co/bitstream/11348/4870/2/20061127153058_El\%20gusano\%20 cogollero\%20del\%20maiz.pdf (consultado 29 oct. 2015).

Neyra, S., y H.E. Chanducas. 2009. Fluctuación poblacional de Spodoptera sp mediante conteo en trampas de saco negro. Rebiol. 29:1-7.

Nexticapan-Garcéz, A., A. Magdub-Méndez, S. Vergara-Yoisura, R. Martín-Mex, y A. Larqué-Saavedra. 2009. Fluctuación poblacional y daños causados por gusano cogollero (Spodoptera frugiperda JE Smith) en maíz cultivado en el sistema de producción continua afectado por el huracán Isidoro. Universidad y Ciencia 25:273-277.

R Development Core Team. 2008. R: A language and environment for statistical computing. R Foundation for Statistical Computing, Vienna, AUS. http://www.R-project.org (consultado 22 ago. 2015).

Rodríguez-Del-Bosque, L.A., and A. Magallanes-Estala. 1994. Seasonal abundance of Diabrotica balteata and other diabroticina beetles (Coleoptera: Chrysomelidae) in northeastern Mexico. Environ. Entomol. 23:1409-1415. doi: 10.1093/ ee/23.6.1409 
Rock, C.G. 1985. Thermal and thermoperiodic effects on larval and pupal development and survival in tufted apple bud moth (Lepidoptera: Tortricidae). Environ. Entomol. 14:637-640. doi: 10.1093/ee/14.5.637

Salas, A.M.D., E. Salazar, y A. Marín. 2002. Manual para la identificación y control de insectos plaga de los cultivos en el Bajío. Universidad de Guanajuato, e Instituto Nacional de Investigaciones Forestales, Agrícoles y Pecuarias, Guanajuato, MEX.

Venette, R.C., D.J. Kriticos, R.D. Magarey, F.H. Koch, R.H.A. Baker, S.P. Worner, N.N. Gómez, D.W. McKenney, E.J. Dobesberger, D. Yemshanov, P.J. De-Barro, W.D. Hutchison, G. Fowler, T.M. Kalaris, and J. Pedlar. 2010. Pest risk maps for invasive alien species: a roadmap for improvement. BioScience 60:349-362.

Villa, C.J., y C.J. Villa. 1996. La ubicación de trampas y factores climáticos afectan el monitoreo de descortezadores en el sur del estado de Jalisco. Cienc. Forestal Méx. 21(79):87-100. 